\title{
Genistein targets the cancerous inhibitor of PP2A to induce growth inhibition and apoptosis in breast cancer cells
}

\author{
QINGXIA ZHAO $^{1,2}$, MING ZHAO $^{2}$, AMANDA B. PARRIS $^{2}$, YING XING $^{1}$ and XIAOHE YANG ${ }^{2}$ \\ ${ }^{1}$ Basic Medical College of Zhengzhou University, Zhengzhou, Henan 450001, P.R. China; \\ ${ }^{2}$ Julius L. Chambers Biomedical/Biotechnology Research Institute and Department of Biology, \\ North Carolina Central University, Kannapolis, NC 28081, USA
}

Received January 25, 2016; Accepted May 30, 2016

DOI: $10.3892 /$ ijo.2016.3588

\begin{abstract}
Genistein is a soy isoflavone with phytoestrogen and tyrosine kinase inhibitory properties. High intake of soy/genistein has been associated with reduced breast cancer risk. Despite the advances in genistein-mediated antitumor studies, the underlying mechanisms remain unclear. In the present study, we investigated genistein-induced regulation of the cancerous inhibitor of protein phosphatase 2A (CIP2A), a novel oncogene frequently overexpressed in breast cancer, and its functional impact on genistein-induced growth inhibition and apoptosis. We demonstrated that genistein induced downregulation of CIP2A in MCF-7-C3 and T47D breast cancer cells, which was correlated with its growth inhibition and apoptotic activities. Overexpression of CIP2A attenuated, whereas CIP2A knockdown sensitized, genistein-induced growth inhibition and apoptosis. We further showed that genistein-induced downregulation of CIP2A involved both transcriptional suppression and proteasomal degradation. In particular, genistein at higher concentrations induced concurrent downregulation of E2F1 and CIP2A. Overexpression of E2F1 attenuated genistein-induced downregulation of CIP2A mRNA, indicating the role of E2F1 in genistein-induced transcriptional suppression of CIP2A. Taken together, our results identified CIP2A as a functional target of genistein and demonstrated that modulation of E2F1-mediated transcriptional regulation of CIP2A contributes to its downregulation. These data advance our understanding of genistein-induced growth inhibition and apoptosis, and support further investigation on CIP2A as a therapeutic target of relevant anticancer agents.
\end{abstract}

Correspondence to: Dr Ying Xing, Basic Medical College of Zhengzhou University, Zhengzhou, Henan 450001, P.R. China E-mail: xingy@zzu.edu.cn

Dr Xiaohe Yang, Julius L. Chambers Biomedical/Biotechnology Research Institute and Department of Biology, North Carolina Central University, Kannapolis, NC 28081, USA

E-mail: xyang@nccu.edu

Key words: genistein, CIP2A, E2F1, cell cycle, apoptosis, breast cancer

\section{Introduction}

Breast cancer is one of the most common cancers threatening women and its global incidence is still increasing. Importantly, breast cancer is the second leading cause of cancer-related deaths in women, which accounts for approximately $15 \%$ of all female cancer-related deaths in the United States (1). Effective prevention and treatment of this morbid disease remain a significant challenge. Use of natural compounds for breast cancer prevention and treatment is one of the major efforts in breast cancer research (2).

Genistein is a major functional component of soy isoflavones. High intake of soy products has been associated with low incidence of breast cancer (3). Soy-associated anticancer activities have been attributed to soy isoflavones (4). Available evidence suggests that genistein is a bioactive molecule with multiple functions, including properties of phytoestrogen, tyrosine kinase and topoisomerase inhibition (5-7). In addition to directly binding to estrogen receptor (ER) (8), receptor tyrosine kinase (RTK) (6) and topoisomerase (7), genistein also modulates a number of key intracellular molecules, such as $\mathrm{NF}-\kappa \mathrm{B}$ and MAPK, to induce growth arrest and apoptosis. Therefore, genistein functions as a multi-targeting antitumor agent (9). Despite these advances, the mechanisms of genistein-mediated tumor inhibition remain unclear. Hence, identification of novel intracellular targets that mediate genistein-induced tumor inhibition is of pivotal significance.

Cancerous inhibitor of protein phosphatase 2A (CIP2A), encoded by the KIAA1524 gene, is a recently identified oncoprotein (10). Overexpression of CIP2A has been detected in tumors from various origins, including breast, lung and colon $(11,12)$, which has been associated with poor prognosis and disease progression $(11,13,14)$. CIP2A expression in breast cancer tissues was also significantly related to higher tumor grades, lymph node and distant metastasis and resistance to chemotherapeutics (15). In vitro expression of CIP2A promotes the immortalization and malignant transformation of human cells (12). CIP2A functions as a protein phosphatase $2 \mathrm{~A}$ (PP2A) inhibitor, a known tumor suppressor $(12,16)$. CIP2A attenuates c-Myc degradation by inhibiting PP2A-mediated dephosphorylation of MYC at serine 62 (13). It was reported that CIP2A and c-Myc formed a positive feed-back loop that promoted the expression of both oncoproteins $(13,17)$. 
Collectively, CIP2A forms an 'oncogenic nexus' to regulate oncogenic transformation of affected cells by virtue of its control of PP2A and MYC stabilization in cancer cells $(18,19)$. Moreover, a number of recent reports indicate that CIP2A not only plays a critical role in tumor development but also functions as a therapeutic target of certain small molecule inhibitors and natural compounds such as erlotinib, celastrol and bortezomib (20-22). Downregulation of CIP2A has been associated with the antitumor activity of these agents.

In the present report, we investigated the role of CIP2A in genistein-treated breast cancer cells. We report here for the first time that genistein induces downregulation of CIP2A, which contributes to genistein-mediated growth inhibition and apoptosis. The underlying mechanisms involve the modulation of E2F1-mediated transcriptional regulation of CIP2A. These results support CIP2A as a molecular target in genisteinmediated tumor inhibition.

\section{Materials and methods}

Reagents and antibodies. Genistein, MG132, RNase A and propidium iodide (PI) were purchased from Sigma-Aldrich. (St. Louis, MO, USA). Antibodies against CIP2A, E2F1 and $\beta$-actin were purchased from Santa Cruz Biotechnology (Santa Cruz, CA, USA). Antibodies against PARP, caspase-3, cleaved caspase-3 and c-Myc were from Cell Signaling Technology (Danvers, MA, USA).

Cell culture, transfection and treatment. MCF-7, T47D and HEK 293T cell lines were obtained from the American Type Culture Collection (ATCC; Rockville, MD, USA). MCF-7caspase-3 (MCF-7-C3) cells were a stable subline of MCF-7 cells which were reconstituted with caspase-3. As described in our previous report (23), this subline was generated by transfecting MCF-7 cells with caspase-3 encoding plasmid followed by antibiotic selections. Puromycin-resistant clones were pooled as a subline for later studies (24). The cells were cultured in DMEM/F12 medium supplemented with $10 \%$ fetal bovine serum (FBS; Atlanta Biologicals, Flowery Branch, GA, USA), $100 \mathrm{U} / \mathrm{ml}$ penicillin and $100 \mathrm{mg} / \mathrm{ml}$ streptomycin at $37^{\circ} \mathrm{C}$ with a $5 \% \mathrm{CO}_{2}$ atmosphere. The cells were treated with genistein or relevant agents as specified in each experiment.

For cell transfection, cells were seeded into a 6 -well plate at $2 \times 10^{5}$ cells/well $24 \mathrm{~h}$ prior to the transfection. $\mathrm{pCMV} / \mathrm{E} 2 \mathrm{~F} 1$ and the control plasmids (25) were transfected into the cells using X-tremeGENE 9 DNA transfection reagent (Roche Disgnostics, Indianapolis, IN, USA) according to the manufacturer's protocol. Twenty-four hours later, the cells were treated with genistein for $48 \mathrm{~h}$ before lysate collection.

Lentivirus production. Lentiviruses encoding CIP2A protein and CIP2A shRNA were prepared from HEK 293T cells by co-transfection of pMD2.G, psPAX2 (Addgene, Cambridge, MA, USA) and specific encoding plasmids with X-tremeGENE 9. pLKO1-CIP2AshRNA and pLKO1/ KIAA1524 were purchased from GeneCopoeia (Rockville, MD, USA). Supernatants from the transfected HEK 293T cells were collected at 24 and $48 \mathrm{~h}$ post-transfection. Supernatants were pooled and filtered through a $0.45 \mu \mathrm{m}$ filter. For lentiviral infection, cells were plated in monolayer at different densities and incubated with lentivirus in the presence of $8 \mu \mathrm{g} / \mathrm{ml}$ polybrene, followed by selection with $1 \mu \mathrm{g} / \mathrm{ml}$ puromycin. Resistant clones were pooled and expanded for later experiments.

Cell proliferation assay. Cell proliferation was assessed with 3-(4,5-dimethylthiazol-2-yl)-2,5-diphenyltetrazolium (MTT) assays. Cells were incubated in 96-well plates at $1 \times 10^{3}$ cells/well $24 \mathrm{~h}$ prior to treatment. The cells were then treated with genistein at indicated concentrations for 5 days, followed by incubation with MTT $(2.5 \mathrm{mg} / \mathrm{ml})$ for $4 \mathrm{~h}$. The medium was then removed and replaced with $50 \mu \mathrm{l} /$ well DMSO, followed by incubation on a shaker for $45 \mathrm{~min}$. Colorimetric absorbance was read with an ELISA reader at $540 \mathrm{~nm}$. Data based on samples of 6 replicates were statistically analyzed.

Western blot analysis. Control or treated cells were collected for lysate preparation as previously described (26). Protein lysates were separated on $10 \%$ SDS-PAGE gels and transferred to a nitrocellulose membrane. The membrane was blocked with $5 \%$ milk in TBS-T buffer for $2 \mathrm{~h}$, followed by incubation with primary antibodies in $5 \%$ BSA-TBS-T at $4^{\circ} \mathrm{C}$ overnight. The membrane was washed with TBS-T and then incubated with corresponding HRP-labeled secondary antibody for $1 \mathrm{~h}$. Protein signals were detected using an ECL detection kit (Thermo Fisher Scientific, Rockford, IL, USA). The images were captured with a FluorChem E system (Cell Biosciences, Santa Clara, CA, USA).

Flow cytometric analysis of cell cycle. Cells were incubated in $60-\mathrm{mm}$ plates $\left(5 \times 10^{5}\right.$ cells/plate) overnight and then treated with genistein at indicated concentrations for $24 \mathrm{~h}$. The cells were harvested to prepare for single cell suspension, followed by drop-wise fixation with $70 \%$ ethanol. Fixed cells were washed with PBS/0.1\% Triton X-100 twice. The cells were then incubated with RNase $\mathrm{A}(1 \mathrm{mg} / \mathrm{ml})$ and propidium iodide $\left(\mathrm{PI}, 33 \mu \mathrm{g} / \mathrm{ml}\right.$ ) at $37^{\circ} \mathrm{C}$ for $45 \mathrm{~min}$, followed analysis with a Guava easyCyte 8 flow cytometer. The percentage of cells in cell cycle phases was analyzed with ModFit program from Verity Software House (Topsham, ME, USA).

Quantitative real-time PCR. Total RNA was extracted from treated cells with an RNeasy Protect Mini kit (Qiagen, Valencia, CA, USA) according to the manufacturer's instructions. Quality and quantity of total RNA were determined by a NanoDrop 1000 Spectrophotometer (Thermo Fisher Scientific, Waltham, MA USA). First-Strand cDNA Synthesis was performed using an iScript cDNA Synthesis kit (Bio-Rad Laboratories, Hercules, CA, USA). Primers targeting CIP2A and GAPDH (glyceraldehydes-3-3phosphate dehydrogenase) were synthesized by Integrated DNA Technologies (Coralville, IA, USA). The sequences for specific primers are: GAPDH-F, 5'-TGC ACC ACC ACC TGC TTA GC-3' and GAPDH-R, 5'-GGC ATG GAC TGT GGT CAT GAG-3'; CIP2A-F, 5'-GAA CAG ATA AGA AAA GAG TTG AGC ATT-3' and CIP2A-R, 5'-CGA CCT TCT AAT TGT GCC TTT T-3' Quantitative real-time PCR reactions were carried out on a CFX96 Real-Time PCR Detection system (Bio-Rad Laboratories). The amplification started with $95^{\circ} \mathrm{C}$ for $10 \mathrm{~min}$, followed by $95^{\circ} \mathrm{C}$ for $15 \mathrm{sec}, 95^{\circ} \mathrm{C}$ for $60 \mathrm{sec}$ for 40 cycles. Relative mRNA levels of CIP2A were normalized to GAPDH levels. 
Apoptosis assay. The Cell Death Detection (ELISA) kit (Roche Life Science, Indianapolis, IN, USA) was used for assessing apoptosis according to the manufacturer's protocol. The cell lysates were incubated in microtiter plate modules coated with anti-histone antibody, followed by washing. Once color developed, samples were measured with a Synergy Mx microplate reader (BioTek Instruments, Inc., Winooski, VT, USA) at $405 \mathrm{~nm}$. All analyses were performed in triplicate.

Statistical analysis. Calculations were performed using software from GraphPad Prism (GraphPad) and data were expressed as means \pm SEM. A two-tailed Student's t-test was used to compare groups. Differences between groups were considered statistically significant at $\mathrm{P}<0.05$.

\section{Results}

Genistein-mediated downregulation of CIP2A is associated with its growth inhibition and apoptosis induction. To determine whether genistein induces CIP2A regulation, we examined CIP2A protein levels in genistein-treated MCF-7, MCF-7-caspase-3 (MCF-7-C3) and T47D breast cancer cells. Because MCF-7 cells are deficient of caspase-3 and do not display typical PARP cleavage and DNA fragmentation (27), we used MCF-7-C3 cells, a stable cell line generated from pooled clones of MCF-7 cells transfected with caspase-3, which have been well characterized in our previous studies $(23,24,28,29)$. As shown in Fig. 1A, when the cells were treated with genistein at concentrations ranging from 0 to $60 \mu \mathrm{M}$ for $48 \mathrm{~h}, \mathrm{CIP} 2 \mathrm{~A}$ protein levels in each cell line were downregulated in a concentration-dependent manner, especially in the 30 and $60 \mu \mathrm{M}$ groups. Importantly, genistein-mediated CIP2A downregulation was associated with growth inhibition and PARP cleavage in MCF-7-C3 and T47D cells that express functional caspase-3. Under similar treatment conditions, genistein also induced inhibition of cell proliferation by MTT assay (Fig. 1B) and cell cycle arrest in G0/G1 phase by flow cytometry (Fig. 1C). Examination of apoptotic markers indicated that genistein-induced downregulation of CIP2A was associated with decreased total caspase-3, and increased cleavage of caspase-3 and PARP (Fig. 1A). Taken together, these results demonstrated that CIP2A is a cellular target of genistein. The association between CIP2A downregulation and increased growth inhibition and apoptosis suggests that CIP2A downregulation may have a functional impact on genistein-induced tumor inhibition.

Overexpression of CIP2A attenuates genistein-induced growth inhibition. To determine whether genistein-induced CIP2A downregulation contributes to its growth inhibition property, we overexpressed CIP2A in MCF-7-C3 and T47D cells and characterized their responses to genistein. By infecting the cells with control and CIP2A encoding lentiviruses followed by puromycin selection, we obtained control (MCF-7-C3/CON and T47D/CON) and CIP2A overexpressing (MCF-7-C3/CIP2A and T47D/CIP2A) stable lines (Fig. 2A). As shown in Fig. 2B, genistein-induced growth inhibition in MCF-7-C3/CIP2A and T47D/CIP2A cells were significantly attenuated as compared to corresponding control cells, indicating that CIP2A is involved in genistein-associated
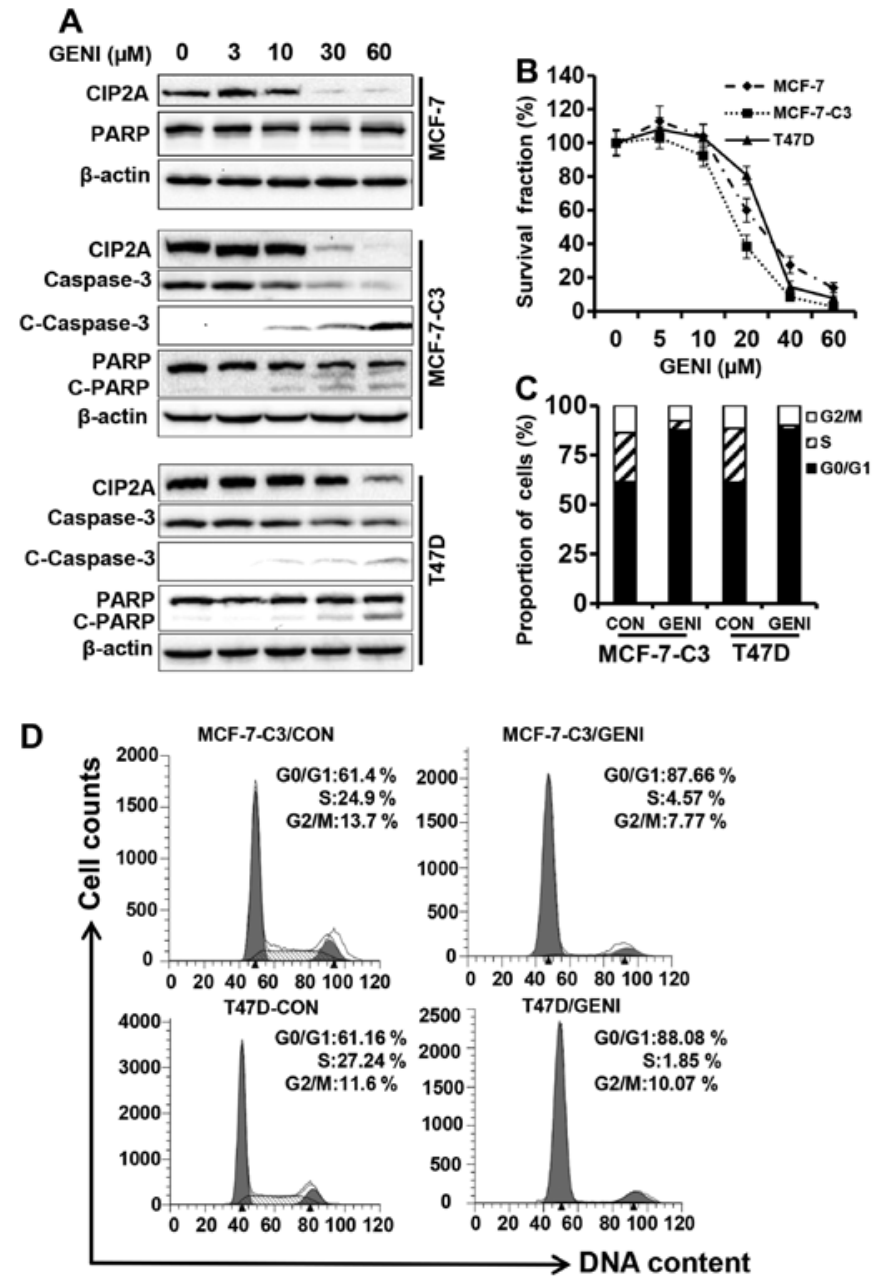

Figure 1. Genistein-mediated downregulation of CIP2A is associated with its growth inhibition and apoptosis induction. (A) MCF-7, MCF-7-C3 and T47D cells were treated with genistein (GENI) at indicated concentrations for $48 \mathrm{~h}$. Protein levels of CIP2A and PARP in all three cell lines, and caspase-3, cleaved caspase-3 (C-caspase-3) in MCF-7-C3 and T47D cells were detected with western blot analysis. (B) Genistein-induced growth inhibition in MCF-7, MCF-7-C3 and T47D cells assessed with MTT assays. The cells in 96-well plates were treated with genistein at indicated concentrations for 5 days, followed by MTT assays as detailed in Materials and methods. The data were based on samples of 6 replicates. (C) Histogram of cell cycle distributions in genistein treated MCF-7-C3 and T47D cells. The cells were treated with $60 \mu \mathrm{M}$ genistein for $24 \mathrm{~h}$, followed by flow cytometric analysis. (D) Cell cycle distributions in different phases were analyzed using ModFit software.

anti-proliferation effect. Cell cycle analysis with flow cytometry indicated that genistein-induced G0/G1 arrest in both MCF-7-C3/CIP2A and T47D/CIP2A cells was decreased as compared to the controls (Fig. 2C).

Overexpression of CIP $2 A$ renders breast cancer cells resistant to genistein-induced apoptosis. To determine the role of CIP2A in genistein-induced apoptosis, we examined apoptotic responses in the two pairs of cell lines in the presence or absence of genistein. Data from apoptosis ELISA assays showed that genistein-induced apoptosis in MCF-7-C3/CIP2A and T47D/CIP2A cells was significantly decreased as compared to MCF-7-C3/CON and T47D/CON cells (Fig. 3A). Consistently, we found that PARP cleavage in genistein-treated MCF-7-C3/CIP2A and T47D/CIP2A cells 
A

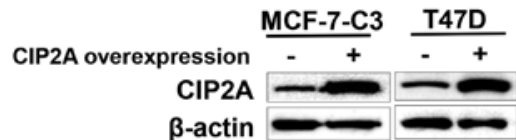

B
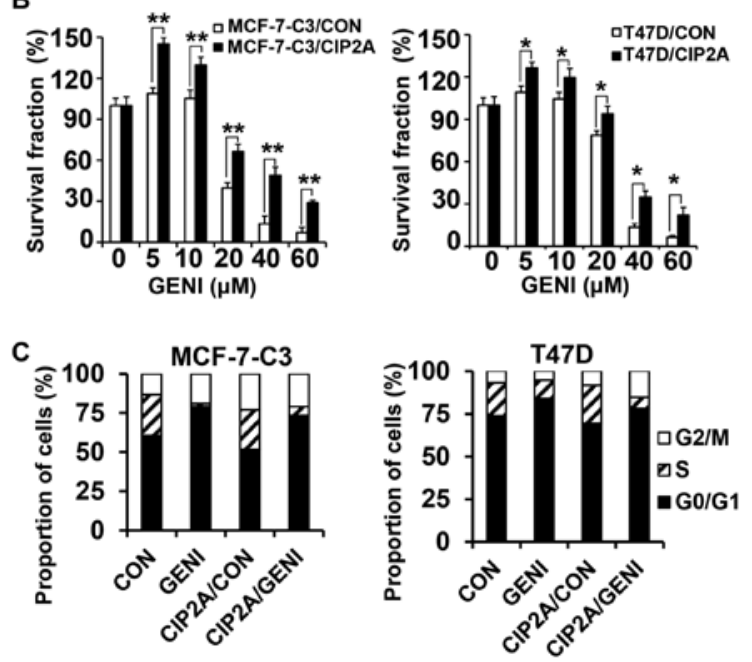

Figure 2. Overexpression of CIP2A attenuates genistein-induced growth inhibition. (A) CIP2A levels in control and CIP2A overexpressing MCF-7-C3 and T47D cells detected with western blot analysis. Cells from each line were infected with control and CIP2A encoding lentiviruses, respectively. Stable CIP2A overexpressing clones were pooled after puromycin selection. (B) CIP2A overexpression attenuates genistein-induced growth inhibition Control and CIP2A overexpressing cells were treated with genistein (GENI) for 5 days, followed by MTT assays. (C) Cell cycle analysis of genisteintreated control and CIP2A overexpressing MCF-7-C3 and T47D cells. The cells were treated with $60 \mu \mathrm{M}$ genistein for $24 \mathrm{~h}$ followed by flow cytometric analysis. ${ }^{*} \mathrm{P}<0.05 ;{ }^{* *} \mathrm{P}<0.01$.

A



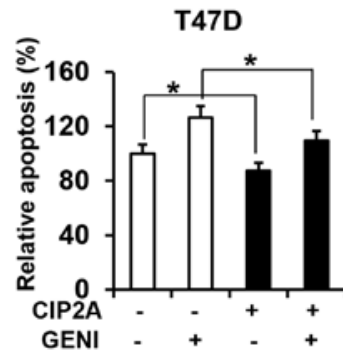

B

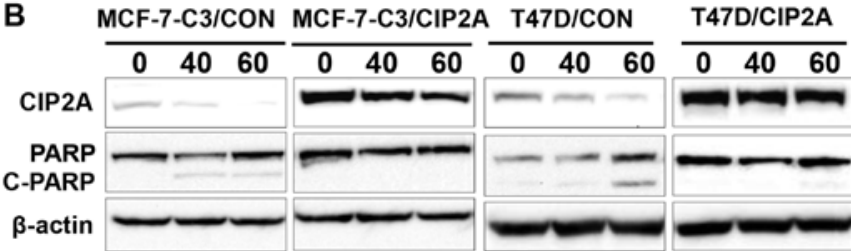

Figure 3. Overexpression of CIP2A reduces genistein-induced apoptosis (A) Control (MCF-7-C3/CON; T47D/CON) and CIP2A overexpressing (MCF-7-C3/CIP2A; T47D/CIP2A) breast cancer cells were treated with $60 \mu \mathrm{M}$ genistein (GENI) for $24 \mathrm{~h}$. Relative apoptosis in individual samples was assessed with apoptosis ELISA assays. The data are presented as mean \pm SEM based on triplicate samples. (B) Control and CIP2A overexpressing MCF-7-C3 and T47D cell lines were treated with genistein at indicated concentrations for $48 \mathrm{~h}$, followed by western blot detection of CIP2A, PARP and $\beta$-actin. ${ }^{*} \mathrm{P}<0.05,{ }^{* * *} \mathrm{P}<0.01$.

was also decreased as compared to the control (Fig. 3B). The data indicate that CIP2A downregulation contributes to genistein-induced apoptosis.

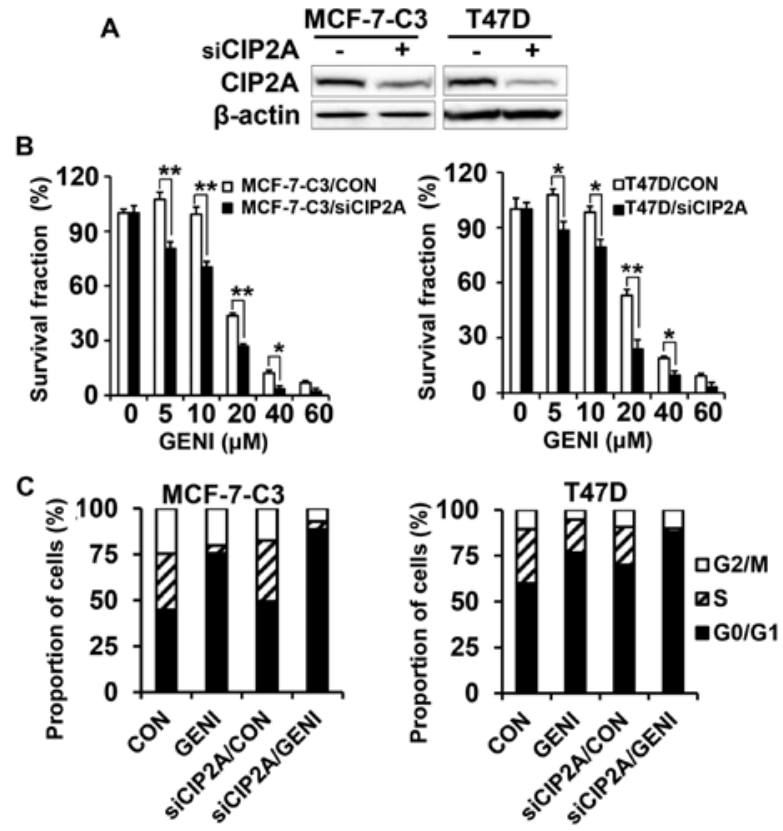

Figure 4. CIP2A knockdown sensitizes MCF-7-C3 and T47D cells to genistein-induced growth inhibition. (A) CIP2A levels in control (siCIP2A -) and CIP2A knockdown (siCIP2A +) cells detected by western blot analysis. MCF-7-C3 and T47D cells were infected with control and CIP2A siRNA encoding lentivirus. Stable clones were collected after puromycin selection. (B) Control and CIP2A knockdown MCF-7-C3 and T47D cells were treated with genistein (GENI) at indicated concentrations for 5 days, followed by MTT assays. (C) Cell cycle analysis of genistein-treated control and CIP2A knockdown MCF-7-C3 and T47D cells. The cells were treated with $60 \mu \mathrm{M}$ genistein for $24 \mathrm{~h}$ followed by flow cytometric analysis. ${ }^{*} \mathrm{P}<0.05 ;{ }^{* *} \mathrm{P}<0.01$.

CIP2A knockdown enhances genistein-induced growth inhibition. To confirm the functional role of CIP2A in genisteininduced growth inhibition and apoptosis, we also established stable MCF-7-C3 and T47D sublines with CIP2A knockdown. As shown in Fig. 4A, CIP2A expression was efficiently knocked down in MCF-7-C3 and T47D cells transfected with CIP2A shRNA encoding lentiviruses. With these paired cell lines, we found that CIP2A knockdown significantly enhanced genisteininduced growth inhibition, as measured with MTT assays (Fig. 4B). Cell cycle analysis showed that CIP2A knockdown alone was able to induce increased G0/G1 arrest. Genisteininduced $\mathrm{G} 0 / \mathrm{G} 1$ arrest was further enhanced in genistein-treated MCF-7-C3/siCIP2A and T47D/siCIP2A cells (Fig. 4C). These results demonstrated that CIP2A plays a role in genisteininduced cell cycle regulation and growth inhibition.

CIP2A knockdown enhances genistein-induced apoptosis. Next, we examined the effect of CIP2A knockdown on genistein-induced apoptosis. The apoptosis ELISA assay showed that genistein induced apoptosis in MCF-7-C3/siCIP2A cells and T47D/siCIP2A cells significantly, as compared to the control (Fig. 5A). Examination of PARP cleavage indicated that genistein induced a significant increase in PARP cleavage in MCF-7-C3/siCIP2A and T47D/siCIP2A cells (Fig. 5B). The data confirm CIP2A's role in genistein-induced apoptosis.

Genistein regulates CIP2A at both $m R N A$ and protein levels. To understand the mechanism of genistein-mediated downregulation of CIP2A, we first examined CIP2A mRNA levels 

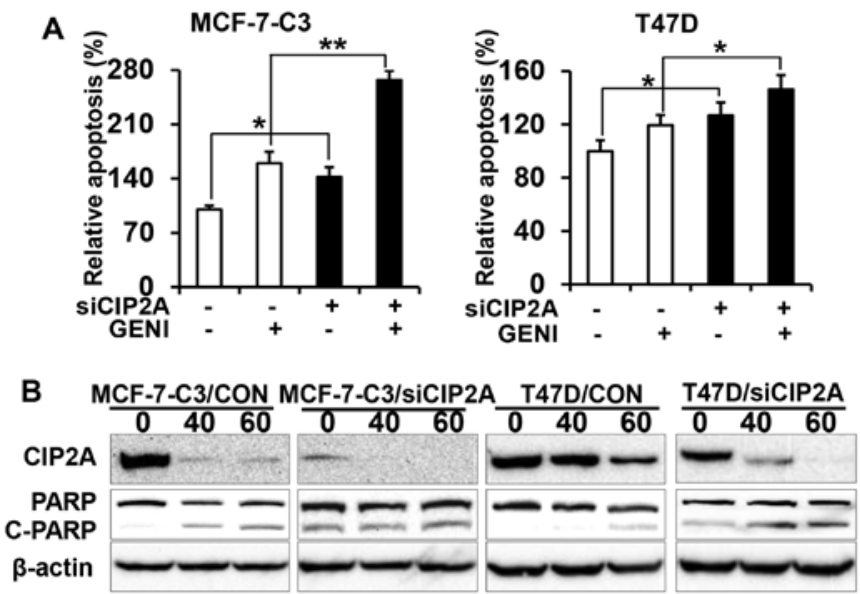

Figure 5. CIP2A knockdown enhances genistein-induced apoptosis. (A) Control (MCF-7-C3/siCON; T47D/siCON) and CIP2A knockdown (MCF-7-C3/siCIP2A; T47D/siCIP2A) cells were treated with $60 \mu \mathrm{M}$ genistein (GENI) for $24 \mathrm{~h}$. Relative apoptosis in individual samples was assessed with apoptosis ELISA assays. The data were presented as mean \pm SEM based on triplicate samples. (B) Control and CIP2A knockdown MCF-7-C3 and T47D cells were treated with genistein at indicated concentrations for $48 \mathrm{~h}$, followed by western blot detection of CIP2A, PARP and $\beta$-actin. ${ }^{*} \mathrm{P}<0.05$, ${ }^{* *} \mathrm{P}<0.01$.
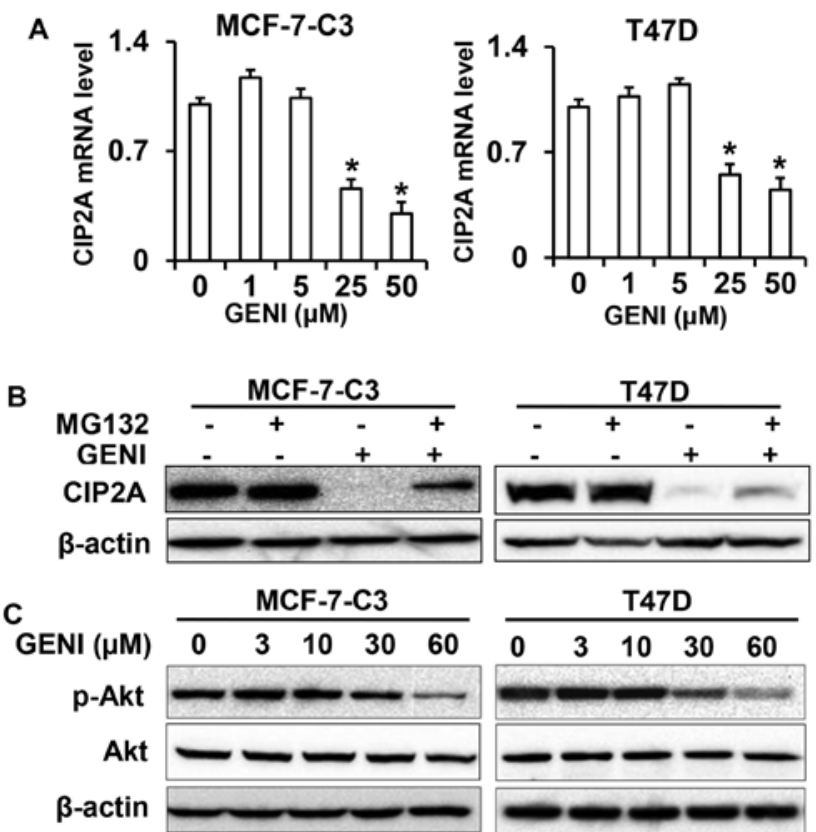

T47D

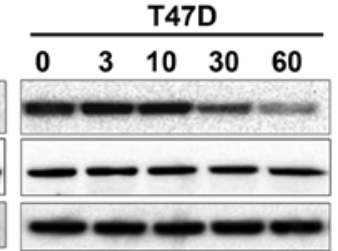

Figure 6. Genistein-induced downregulation of CIP2A involves both transcriptional and post-translational regulation. (A) CIP2A mRNA levels in control and genistein (GENI) treated MCF-7-C3 and T47D cells. The cells were treated with genistein at indicated concentrations for $16 \mathrm{~h}$. Relative CIP2A mRNA levels were detected with quantitative RT-PCR. mRNA levels of GAPDH were used for normalization. (B) MG132 attenuates genistein-induced downregulation of CIP2A. MCF-7-C3 and T47D cells were treated with $60 \mu \mathrm{M}$ genistein in the absence (-) or presence (+) of MG132 $(1 \mu \mathrm{M})$ for $24 \mathrm{~h}$, followed by western blot detection of CIP2A and $\beta$-actin. (C) Genistein inhibits Akt phosphorylation. MCF-7-C3 and T47D cells were treated with genistein as in Fig. 1A. Phospho-Akt and Akt were detected with western blotting. ${ }^{~} \mathrm{P}<0.05$.

in genistein-treated MCF-7-C3 and T47D cells. As shown in Fig. 6A, CIP2A mRNA levels in the cells treated with 25 or $50 \mu \mathrm{M}$ genistein were significantly decreased. Notably, CIP2A mRNA levels in the cells treated with genistein at

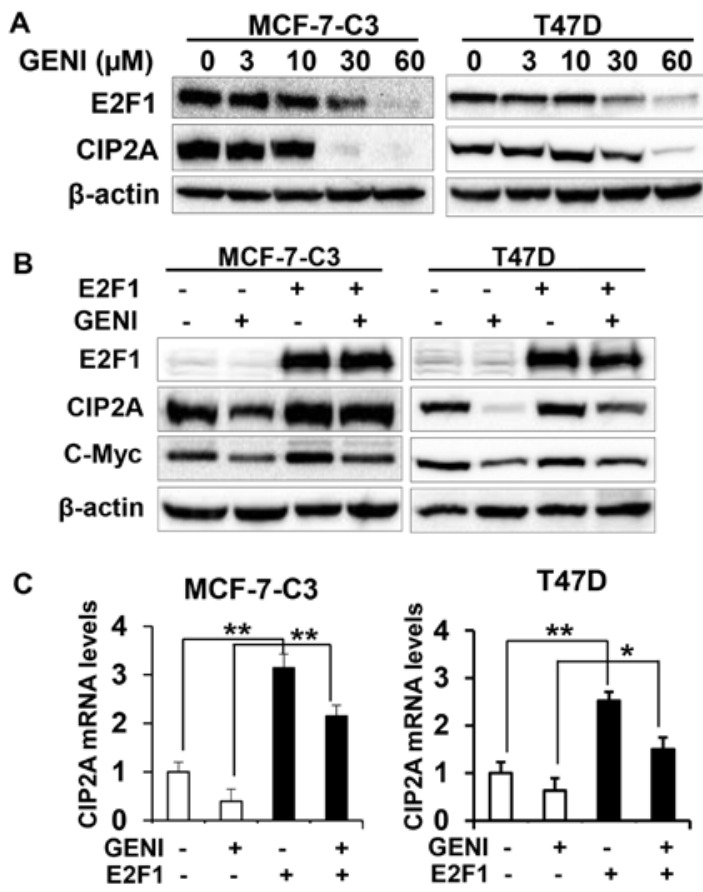

Figure 7. Genistein-induced downregulation of CIP2A involves the modulation of E2F1-mediated CIP2A transcription. (A) Genistein induces concurrent downregulation of E2F1 and CIP2A. MCF-7-C3 and T47D cells were treated with genistein (GENI) for $48 \mathrm{~h}$. Protein levels of CIP2A and E2F1 were detected with western blot analysis. (B) Overexpression of E2F1 attenuates genistein-induced CIP2A downregulation. MCF-7-C3 and T47D cells were transiently transfected with control (-) and E2F1 encoding (+) plasmids. Twenty-four hours post-transfection, the cells were treated with $60 \mu \mathrm{M}$ genistein for $48 \mathrm{~h}$, followed by western blot detection of E2F1, CIP2A, c-Myc and actin. (C) CIP2A mRNA levels in control and E2F1 overexpressing MCF-7/C3 and T47D cells with different treatments. The cells were transiently transfected with control and E2F1 encoding plasmids followed by genistein $(60 \mu \mathrm{M})$ treatment for $16 \mathrm{~h}$. Relative CIP2A mRNA levels were detected with quantitative RT-PCR. mRNA levels of GAPDH were used for normalization. ${ }^{*} \mathrm{P}<0.05,{ }^{* *} \mathrm{P}<0.01$

lower concentrations were slightly increased. This dose-effect pattern was consistent with the protein level changes observed above. These results indicate that genistein-mediated inhibition of CIP2A transcription is involved in its downregulation of CIP2A.

As it was reported that CIP2A could be regulated by proteasomal degradation in celastrol treated cancer cells (21), we then examined whether modulation of proteasomal degradation contributes to genistein-mediated downregulation of CIP2A. We found that treating the cells with proteasomal inhibitor MG132 significantly reversed genistein-induced downregulation of CIP2A, suggesting that genistein also regulates CIP2A at the protein level (Fig. 6B). As CIP2A protein stability could be regulated by Akt activation (21), we examined phospho-Akt levels in genistein-treated cells (Fig. 6C). Inhibition of phospho-Akt in the treated cells suggests a link between these changes and CIP2A protein degradation.

Genistein-induced downregulation of CIP2A involves E2F1mediated transcriptional regulation. To further investigate the underlying mechanism of genistein-induced downregulation of CIP2A, we focused on genistein-induced transcriptional downregulation. It was reported that E2F1 is a major transcription factor that regulates CIP2A transcription $(30,31)$. 
We therefore examined the role of $\mathrm{E} 2 \mathrm{~F} 1$ in genistein-mediated transcriptional regulation of CIP2A. As shown in Fig. 7A, genistein-induced concurrent decrease of CIP2A and E2F1 protein levels in both MCF-7-C3 and T47D cells, suggesting a correlation between the two molecules. To define the specific role of E2F1, we examined the effect of E2F1 overexpression on genistein-modulated CIP2A mRNA and protein levels. We found that E2F1 overexpression attenuated genistein-induced downregulation of CIP2A protein (Fig. 7B). Examination of mRNA levels in the cells with different E2F1 status and genistein treatments indicated that $\mathrm{E} 2 \mathrm{~F} 1$ overexpression induced a dramatic increase in CIP2A mRNA, whereas genisteininduced downregulation of CIP2A mRNA was abolished in both cell lines with E2F1 overexpression (Fig. 7C). The results suggest that E2F1 plays a critical role in genistein-mediated downregulation of CIP2A mRNA, which contribute to the overall downregulation of CIP2A protein.

\section{Discussion}

In the present study, we demonstrated that genistein induced downregulation of CIP2A in MCF-7-C3 and T47D breast cancer cells, which was associated with its growth inhibition and apoptosis induction. We further demonstrated that overexpression of CIP2A attenuated genistein-induced growth inhibition and apoptosis, and conversely, CIP2A knockdown sensitized genistein-induced cellular effects. Our results indicate that genistein specifically regulates CIP2A in breast cancer cells and the consequent CIP2A downregulation may play a critical role in its antitumor effects. This study identifies CIP2A as a novel cellular target of genistein. Although previous studies have established ER, EGFR and topoisomerase as the major targets of genistein-mediated inhibition in breast cancer cells (5-7), the intracellular mediators of genistein-associated cellular activities remain obscure. Although CIP2A might not be a direct binding target of genistein, it is a critical intracellular target/mediator of genistein. This study is an advancement in the understanding of genistein-mediated growth inhibition and apoptosis.

The functional impact of genistein-mediated CIP2A downregulation is supported by its correlation with genisteininduced growth inhibition and apoptosis. We showed that CIP2A overexpression reduced genistein-induced overall growth inhibition, cell cycle arrest in G0/G1 phase and apoptosis in each cell line. In contrast, CIP2A knockdown enhanced genistein-induced cell cycle arrest and apoptosis. These data demonstrated the specific role of CIP2A modulation in genistein-associated tumor inhibition. Previously, it was reported that genistein could induce $\mathrm{G} 2 / \mathrm{M}(32,33)$ or G0/G1 arrest (34-37). Under the conditions in the present study, CIP2A modulation was a critical factor affecting genistein-induced G0/G1 arrest. As an oncogene, CIP2A overexpression or knockdown alone could increase and reduce $\mathrm{S}$ phase cells (19), respectively, as it was observed in T47D cells in this study (Figs. 2D and 4D). Although CIP2A overexpression or knockdown modified genistein-induced cell cycle progression, the differences between non-genistein treated control $(\mathrm{CON})$ and $\mathrm{CIP} 2 \mathrm{~A} /$ control $(\mathrm{CIP} 2 \mathrm{~A} / \mathrm{CON})$ cells were not evident (Figs. 2C and 4C). This could be because these cells were stable lines derived from pooled resistant clones, the cells might have developed adaptations to the new environment under non-stress conditions. Nevertheless, the effect of CIP2A modulation in genistein-treated control and the overexpressing/knockdown cells was evident.

Our findings on genistein-mediated downregulation of CIP2A open new avenues to understand genistein-mediated antitumor effects. Increasing evidence has indicated that CIP2A is a pivotal molecule involved in cancer development and progression $(12,13,38)$. It acts as an endogenous inhibitor of PP2A and has a broad impact on cellular proliferation and survival (12,39-41). Deregulation of CIP2A leads to the upregulation/activation of Myc and Akt $(42,43)$. Indeed, our recent data indicate the existence of a positive CIP2A-Akt feedback loop (Zhao et al, unpublished data). Whether genistein directly acts on Akt kinase activity is an intriguing question to be addressed in future studies. Given the critical role of CIP2A in the regulation of the PP2A network, genistein-induced downregulation of CIP2A would inhibit these pathways and contribute to its overall inhibition of cancer cells. This is supported by our data showing that CIP2A overexpression or knockdown resulted in significant changes in genistein-induced cell cycle progression, growth inhibition and apoptosis. Moreover, our results also support CIP2A as a therapeutic target as it was reported in the studies on other anticancer agents $(44,45)$.

Our results showed that genistein induced downregulation of CIP2A mRNA levels, whereas proteasomal inhibitor MG132 treatment also attenuated genistein-induced downregulation of CIP2A protein, indicating that genistein regulates CIP2A expression at both transcriptional and post-translational levels. Previously, induction of CIP2A degradation through proteasomal pathways in response to celastrol has been reported (21). This could be triggered by direct activation of E3 ligase such as CHIP or through inactivation of Akt (21). Based on our data that genistein inhibited Akt phosphorylation (Fig. 6C), it is possible that genistein-induced inhibition of Akt activation at higher concentrations may contribute to this process. Notably, it was reported that genistein may function as a proteasomal inhibitor to induce p27 and Bax in SV-40 transformed cells (46), suggesting that genistein-induced proteasomal regulation is complicated and multi-level, multifaceted mechanisms may exist. Whether genistein has direct effect on E3 ligase activities and whether genistein directly binds and inhibits Akt will be investigated in future studies.

We explored the mechanisms of genistein-mediated inhibition of CIP2A transcription by focusing on E2F1-mediated regulation of CIP2A. E2F1 is a critical regulator of cell cycle progression (47), although it may induce apoptosis under certain cellular contexts (48). It was reported that E2F1 is a major transcription factor that promotes CIP2A expression (49), and CIP2A has a positive feedback effect on E2F1 by stabilizing serine 364 phosphorylation of E2F1 (49). We showed that genistein-induced concurrent downregulation of E2F1 and CIP2A at higher concentrations. Importantly, overexpression of E2F1 attenuated genistein-induced downregulation of CIP2A protein and mRNA levels as well. These data indicate that modulation of E2F1-mediated regulation of CIP2A transcription and E2F1-CIP2A feedback loop is involved in genistein-induced downregulation of CIP2A. While the immediate mediators between genistein treatment 
and E2F1 downregulation require further investigation, the regulation may be determined by the functional interaction among E2F1, c-Myc and CIP2A, a mutual interacting multifactorial network. Recently, it was proposed that CIP2A promotes carcinogenesis via an 'oncogenic nexus', which involves its intersection with PP2A, Akt, Myc and other factors $(18,19)$. Hence, our data suggest that genistein-mediated tumor inhibition involves the suppression of the 'oncogenic nexus' of CIP2A.

The present study provided proof of concept for CIP2A downregulation in genistein-induced inhibition of breast cancer cells. However, how genistein regulates CIP2A degradation and transcription appears to be complicated. More work is needed to understand genistein-mediated regulation of proteasomal pathways in CIP2A degradation. Genisteininduced inhibition of CIP2A transcription may also involve the regulation of other mechanisms. For example, we observed that genistein may induce CIP2A mRNA increases at lower concentrations. This might be mediated by its estrogenic activity, because it was reported that activation of ER also promotes CIP2A expression (50). Although genistein-induced inhibition of CIP2A transcription at higher concentrations may also be related to genistein-associated anti-estrogenic activity at higher concentrations, genistein-induced downregulation of E2F1 and the consequent CIP2A mRNA decrease could be independent of estrogen signaling, as it was also observed in ER negative MDA-MB-231 breast cancer cells (data not shown). Further analysis of the relative contributions of individual mechanisms in genistein-mediated downregulation of CIP2A will be followed in future studies.

In conclusion, our results identified CIP2A as an intracellular target of genistein, which has a functional impact on genistein-induced growth inhibition and apoptosis induction. Genistein-mediated downregulation of CIP2A involves both transcriptional and proteasomal regulation. While multiple factors might contribute to genistein-mediated transcriptional downregulation of CIP2A, we demonstrated that modulation of E2F1-mediated CIP2A transcription plays a critical role in genistein-induced cellular responses. While being consistent with previous reports (31), these data also advance our understanding of genistein-associated antitumor activities and support its implication in breast cancer prevention and treatment. The study also supports the previous implication of CIP2A as a therapeutic target in the development of novel anticancer agents (51).

\section{Acknowledgements}

The present study was supported in part by a grant from NIEHS (R21ES025337) and a pilot project of a U54 grant from NCI (5U54CA156735). X.Y. is also supported by a grant from American Cancer Society (RSG-08-138-01-CNE) and a pilot project grant of the NIH U54 AA019765 grant. We thank Dr Erin Witalison for assistance in manuscript editing.

\section{References}

1. Ferlay J1, Soerjomataram I, Dikshit R, Eser S, Mathers C, Rebelo M, Parkin DM, Forman D and Bray F: Cancer incidence and mortality worldwide: sources, methods and major patterns in GLOBOCAN 2012. Int J Cancer 136: E359-386, 2015.
2. Bonofiglio D, Giordano C, De Amicis F, Lanzino M and Ando S: Natural products as promising antitumoral agents in breast cancer: Mechanisms of action and molecular targets. Mini Rev Med Chem 15: 1, 2015.

3. Nagata C, Mizoue T, Tanaka K, Tsuji I, Tamakoshi A, Matsuo K, Wakai K, Inoue M, Tsugane S, Sasazuki S, et al; Research Group for the Development and Evaluation of Cancer Prevention Strategies in Japan: Soy intake and breast cancer risk: An evaluation based on a systematic review of epidemiologic evidence among the Japanese population. Jpn J Clin Oncol 44: 282-295, 2014.

4. Lamartiniere CA: Protection against breast cancer with genistein: a component of soy. Am J Clin Nutr 71: 1705S-1707S; discussion 1708S-1709S, 2000.

5. Shao ZM, Shen ZZ, Fontana JA and Barsky SH: Genistein's 'ER-dependent and independent' actions are mediated through ER pathways in ER-positive breast carcinoma cell lines. Anticancer Res 20: 2409-2416, 2000.

6. Akiyama T, Ishida J, Nakagawa S, Ogawara H, Watanabe S, Itoh N, Shibuya M and Fukami Y: Genistein, a specific inhibitor of tyrosine-specific protein kinases. J Biol Chem 262: 5592-5595, 1987.

7. Corbett AH, Hong D and Osheroff N: Exploiting mechanistic differences between drug classes to define functional drug interaction domains on topoisomerase II. Evidence that several diverse DNA cleavage-enhancing agents share a common site of action on the enzyme. J Biol Chem 268: 14394-14398, 1993

8. Wang TT, Sathyamoorthy N and Phang JM: Molecular effects of genistein on estrogen receptor mediated pathways. Carcinogenesis 17: 271-275, 1996.

9. Sarkar FH and Li Y: Mechanisms of cancer chemoprevention by soy isoflavone genistein. Cancer Metastasis Rev 21: 265-280, 2002 .

10. Khanna A, Pimanda JE and Westermarck J: Cancerous inhibitor of protein phosphatase $2 \mathrm{~A}$, an emerging human oncoprotein and a potential cancer therapy target. Cancer Res 73: 6548-6553, 2013.

11. Côme C1, Laine A, Chanrion M, Edgren H, Mattila E, Liu X, Jonkers J, Ivaska J, Isola J, Darbon JM, et al: CIP2A is associated with human breast cancer aggressivity. Clin Cancer Res 15: 5092-5100, 2009.

12. Junttila MR, Puustinen $P$, Niemelä $M$, Ahola R, Arnold $H$, Böttzauw T, Ala-aho R, Nielsen C, Ivaska J, Taya Y, et al: CIP2A inhibits PP2A in human malignancies. Cell 130: 51-62, 2007.

13. Khanna A, Böckelman C, Hemmes A, Junttila MR, Wiksten JP, Lundin M, Junnila S, Murphy DJ, Evan GI, Haglund C, et al: MYC-dependent regulation and prognostic role of CIP2A in gastric cancer. J Natl Cancer Inst 101: 793-805, 2009.

14. Khanna A and Pimanda JE: Clinical significance of cancerous inhibitor of protein phosphatase $2 \mathrm{~A}$ in human cancers. Int $\mathrm{J}$ Cancer 138: 525-532, 2016.

15. Yu G, Liu G, Dong J and Jin Y: Clinical implications of CIP2A protein expression in breast cancer. Med Oncol 30: 524, 2013.

16. Mumby M: PP2A: Unveiling a reluctant tumor suppressor. Cell 130: 21-24, 2007.

17. Mathiasen DP, Egebjerg C, Andersen SH, Rafn B, Puustinen P, Khanna A, Daugaard M, Valo E, Tuomela S, Bøttzauw T, et al: Identification of a c-Jun N-terminal kinase-2-dependent signal amplification cascade that regulates c-Myc levels in ras transformation. Oncogene 31: 390-401, 2012.

18. De P, Carlson J, Leyland-Jones B and Dey N: Oncogenic nexus of cancerous inhibitor of protein phosphatase 2A (CIP2A): An oncoprotein with many hands. Oncotarget 5: 4581-4602, 2014.

19. De P, Carlson JH, Leyland-Jones B and Dey N: Role of 'oncogenic nexus' of CIP2A in breast oncogenesis: How does it work? Am J Cancer Res 5: 2872-2891, 2015.

20. Chao TT, Wang CY, Lai CC, Chen YL, Tsai YT, Chen PT, Lin HI, Huang YC, Shiau CW, Yu CJ, et al: TD-19, an erlotinib derivative, induces epidermal growth factor receptor wild-type nonsmall-cell lung cancer apoptosis through CIP2A-mediated pathway. J Pharmacol Exp Ther 351: 352-358, 2014.

21. Liu Z, Ma L, Wen ZS, Hu Z, Wu FQ, Li W, Liu J and Zhou GB: Cancerous inhibitor of PP2A is targeted by natural compound celastrol for degradation in non-small-cell lung cancer. Carcinogenesis 35: 905-914, 2014.

22. Ding Y, Wang Y, Ju S, Wu X, Zhu W, Shi F and Mao L: Role of CIP2A in the antitumor effect of bortezomib in colon cancer. Mol Med Rep 10: 387-392, 2014.

23. Yang XH, Edgerton S and Thor AD: Reconstitution of caspase-3 sensitizes MCF-7 breast cancer cells to radiation therapy. Int J Oncol 26: 1675-1680, 2005. 
24. Yang XH, Sladek TL, Liu X, Butler BR, Froelich CJ and Thor AD: Reconstitution of caspase 3 sensitizes MCF-7 breast cancer cells to doxorubicin- and etoposide-induced apoptosis. Cancer Res 61: 348-354, 2001.

25. Yang XH and Sladek TL: Overexpression of the E2F-1 transcription factor gene mediates cell transformation. Gene Expr 4: 195-204, 1995.

26. Yang X, Yang S, McKimmey C, Liu B, Edgerton SM, Bales W, Archer LT and Thor AD: Genistein induces enhanced growth promotion in ER-positive/erbB-2-overexpressing breast cancers by ER-erbB-2 cross talk and p27/kipl downregulation. Carcinogenesis 31: 695-702, 2010.

27. Jänicke RU, Sprengart ML, Wati MR and Porter AG: Caspase-3 is required for DNA fragmentation and morphological changes associated with apoptosis. J Biol Chem 273: 9357-9360, 1998.

28. Talanian RV, Yang X, Turbov J, Seth P, Ghayur T, Casiano CA, Orth K and Froelich CJ: Granule-mediated killing: Pathways for granzyme B-initiated apoptosis. J Exp Med 186: 1323-1331, 1997.

29. Yang S, Thor AD, Edgerton S and Yang X: Caspase-3 mediated feedback activation of apical caspases in doxorubicin and TNF-alpha induced apoptosis. Apoptosis 11: 1987-1997, 2006.

30. Zhang W, Chen H, Chen Y, Liu J, Wang X, Yu X, Chen JJ and Zhao W: Cancerous inhibitor of protein phosphatase $2 \mathrm{~A}$ contributes to human papillomavirus oncoprotein E7-induced cell proliferation via E2F1. Oncotarget 6: 5253-5262, 2015.

31. Laine A, Sihto H, Come C, Rosenfeldt MT, Zwolinska A, Niemelä M, Khanna A, Chan EK, Kähäri VM, KellokumpuLehtinen PL, et al: Senescence sensitivity of breast cancer cells is defined by positive feedback loop between CIP2A and E2F1. Cancer Discov 3: 182-197, 2013.

32. Constantinou AI, Kamath N and Murley JS: Genistein inactivates bcl-2, delays the $\mathrm{G} 2 / \mathrm{M}$ phase of the cell cycle, and induces apoptosis of human breast adenocarcinoma MCF-7 cells. Eur J Cancer 34: 1927-1934, 1998

33. Oki T, Sowa Y, Hirose T, Takagaki N, Horinaka M, Nakanishi R, Yasuda C, Yoshida T, Kanazawa M, Satomi Y, et al: Genistein induces Gadd45 gene and G2/M cell cycle arrest in the DU145 human prostate cancer cell line. FEBS Lett 577: 55-59, 2004

34. Yu JY, Lee JJ, Lim Y, Kim TJ, Jin YR, Sheen YY and Yun YP. Genistein inhibits rat aortic smooth muscle cell proliferation through the induction of p27 $7^{\text {kipl }}$. J Pharmacol Sci 107: 90-98, 2008 .

35. Kuzumaki T, Kobayashi T and Ishikawa K: Genistein induces $\mathrm{p} 21^{\mathrm{Cip} 1 / \mathrm{WAF} 1}$ expression and blocks the $\mathrm{G} 1$ to $\mathrm{S}$ phase transition in mouse fibroblast and melanoma cells. Biochem Biophys Res Commun 251: 291-295, 1998.

36. Shen JC, Klein RD, Wei Q, Guan Y, Contois JH, Wang TT, Chang S and Hursting SD: Low-dose genistein induces cyclindependent kinase inhibitors and $\mathrm{G}_{1}$ cell-cycle arrest in human prostate cancer cells. Mol Carcinog 29: 92-102, 2000.

37. Yang $S$, Zhou $Q$ and Yang X: Caspase- 3 status is a determinant of the differential responses to genistein between MDA-MB-231 and MCF-7 breast cancer cells. Biochim Biophys Acta 1773: 903-911, 2007
38. Guo Z, Liu D and Su Z: CIP2A mediates prostate cancer progression via the c-MYC signaling pathway. Tumour Biol 36: $3583-3589,2015$

39. Kim JS, Kim EJ, Oh JS, Park IC and Hwang SG: CIP2A modulates cell-cycle progression in human cancer cells by regulating the stability and activity of Plk1. Cancer Res 73: 6667-6678, 2013

40. Ventelä S, Côme C, Mäkelä JA, Hobbs RM, Mannermaa L, Kallajoki M, Chan EK, Pandolfi PP, Toppari J and Westermarck J: CIP2A promotes proliferation of spermatogonial progenitor cells and spermatogenesis in mice. PLoS One 7: e33209, 2012.

41. Wang J, Huang T, Sun J, Yu Y, Liu Z, Li W, Jia J and Chen C: CIP2A is overexpressed and involved in the pathogenesis of chronic myelocytic leukemia by interacting with breakpoint cluster region-Abelson leukemia virus. Med Oncol 31: 112, 2014

42. Lei N, Peng B and Zhang JY: CIP2A regulates cell proliferation via the AKT signaling pathway in human lung cancer. Oncol Rep 32: 1689-1694, 2014.

43. Niemelä M, Kauko O, Sihto H, Mpindi JP, Nicorici D, Pernilä P, Kallioniemi OP, Joensuu H, Hautaniemi S and Westermarck J: CIP2A signature reveals the MYC dependency of CIP2Aregulated phenotypes and its clinical association with breast cancer subtypes. Oncogene 31: 4266-4278, 2012.

44. Khanna A, Rane JK, Kivinummi KK, Urbanucci A,Helenius MA, Tolonen TT, Saramäki OR, Latonen L, Manni V, Pimanda JE, et al: CIP2A is a candidate therapeutic target in clinically challenging prostate cancer cell populations. Oncotarget 6: 19661-19670, 2015.

45. Gonzalez-Alonso P, Cristobal I, Manso R, Madoz-Gurpide J, Garcia-Foncillas J and Rojo F: PP2A inhibition as a novel therapeutic target in castration-resistant prostate cancer. Tumour Biol 36: $5753-5755,2015$

46. Kazi A, Daniel KG, Smith DM, Kumar NB and Dou QP: Inhibition of the proteasome activity, a novel mechanism associated with the tumor cell apoptosis-inducing ability of genistein. Biochem Pharmacol 66: 965-976, 2003.

47. Mundle SD and Saberwal G: Evolving intricacies and implications of E2F1 regulation. FASEB J 17: 569-574, 2003.

48. Moon NS, Frolov MV, Kwon EJ, Di Stefano L, Dimova DK, Morris EJ, Taylor-Harding B, White K and Dyson NJ: Drosophila E2F1 has context-specific pro- and antiapoptotic properties during development. Dev Cell 9: 463-475, 2005.

49. Laine A and Westermarck J: Molecular pathways: harnessing E2F1 regulation for prosenescence therapy in p53-defective cancer cells. Clin Cancer Res 20: 3644-3650, 2014.

50. Choi YA, Koo JS, Park JS, Park MY, Jeong AL, Oh KS and Yang Y: Estradiol enhances CIP2A expression by the activation of p70 S6 kinase. Endocr Relat Cancer 21: 189-202, 2014.

51. Xue Y, Wu G, Wang X, Zou X, Zhang G, Xiao R, Yuan Y, Long D, Yang J, Wu Y, et al: CIP2A is a predictor of survival and a novel therapeutic target in bladder urothelial cell carcinoma. Med Oncol 30: 406, 2013. 